\section{Fatores que interferem na transição alimentar de crianças entre cinco e oito meses: investigação em Serviço de Puericultura do Recife, Brasil}

\section{Factors that interfere in the food transition in children aged between five and eight months: an investigation of the childcare system in Recife, Brazil}

Carmina Silva dos Santos ${ }^{1}$

Luciane Soares de Lima ${ }^{2}$

Marly Javorski ${ }^{3}$

1 Instituto Materno Infantil Professor Fernando Figueira - IMIP Recife, PE, Brasil. CEP: 50.070-550.

2,3 Departamento de Enfermagem. Universidade Federal de Pernambuco. Av. Prof. Moraes Rego s.n. Cidade Universitária. Recife, PE, Brasil. E-mail: lucianelima@globo.com

\begin{abstract}
Objectives: to identify five- and eight-month old breast-feeding infants fed according to Health Ministry recommendations, cared for by the Instituto Materno Infantil Professor Fernando Figueira - IMIP Childcare Service; to ascertain the major problems faced by mothers in the process of weaning children; to identify the type of guidance given to mothers on how to initiate the food transition.
\end{abstract}

Methods: a descriptive, cross-sectional study was carried out involving 101 mothers/children aged between five- and eight-months who had initiated food transition between April and August 2003. The mothers were asked to participate in the study prior to a consultation with a physician, according to criteria for inclusion and exclusion. The children were classified in compliance with the norms established by the Ministry of Health regarding the food transition.

Results: it was found that $79.2 \%$ of the children did not meet the Ministry of Health's norms for food transition. The reasons for not complying with the norms mentioned by mothers were as follows: refusal on the part of the child (23.5\%); interference of grandmothers (19\%); practicality of preparation/provision of porridge compared with solid food (17.7\%). As for the type of guidance, $97.4 \%$ claimed to have received spoken and written guidance at work. In the case of the sample studied, nurses and grandmothers had played a major role in providing guidance on the food transition, $77 \%$ and $16 \%$, respectively.

Conclusion: only $20.8 \%$ of the children displayed an adequate feeding standard complying with the norms of the Ministry of Health. The mother, who is the one usually responsible for child care, was influenced by family members, especially the grandmother, and by society at large. It was also found that the majority (97.4\%) of the mothers received written as well as spoken guidance.

Key words Transition food, Mothers, Puericulture, Breastfeeding infants

\section{Resumo}

Objetivos: identificar os lactentes de cinco a oito meses alimentados segundo as recomendações do Ministério da Saúde, atendidos no Serviço de Puericultura do Instituto Materno Infantil Professor Fernando Figueira - IMIP; detectar os principais problemas enfrentados pelas mães no processo de introdução alimentar; identificar o tipo de orientação recebida pelas mães para iniciar a dieta de transição.

Métodos: estudo transversal, descritivo, formado por 101 mães de crianças entre cinco e oito meses que haviam iniciado transição alimentar no período de abril a agosto de 2003, as quais foram solicitadas a participar do estudo antes da consulta, mediante os critérios de inclusão e exclusão. As crianças foram classificadas quanto à adequação às normas do Ministério da Saúde para transição alimentar.

Resultados: constatou-se que 79,2\% dessas crianças não se adequavam às normas do Ministério da Saúde quanto à transição alimentar. Para o não seguimento a essas normas as mães apontaram, como principais motivos: recusa da criança $(23,5 \%)$; interferência da avó (19\%); praticidade do preparoloferecimento do mingau em relação à alimentação salgada (17,7\%). Quanto ao tipo de orientação, 97,4\% afirmaram receber as orientações verbais e escritas no serviço. Na amostra estudada o enfermeiro e a avó foram os principais orientadores da transição alimentar, com $77 \%$ e $16 \%$, respectivamente.

Conclusões: apenas 20,8\% das crianças apresentaram um padrão alimentar adequado, segundo as orientações do Ministério da Saúde. A mãe, principal responsável pelos cuidados da criança, sofreu influências do seu ambiente familiar, sobretudo da avó, e da sociedade em que está inserida. Também foi observado que, para a transição alimentar das crianças, 97,4\% das mães recebeu orientação escrita além da orientação verbal.

Palavras-chave Alimentação de transição, Mães, Puericultura, Lactente 


\section{Introdução}

Durante os primeiros seis meses de vida o leite materno é o alimento capaz de atender, de forma completa, todas as peculiaridades fisiológicas do metabolismo dos lactentes. Acresce ainda que o contato físico entre mãe e filho, durante a amamentação, proporciona a transferência de carinho e afeto, bilateralmente, e deve ser valorizado tanto quanto os nutrientes do leite. Ao envolver uma criança para ser amamentada, mãe e filho se enlaçam afetivamente, o que os alimenta reciprocamente, também do ponto de vista psicológico. ${ }^{1}$

Após este período, faz-se necessária a introdução, de forma gradual, de alimentos complementares que atendam as necessidades nutricionais da criança. Essa introdução alimentar, assim como o aleitamento materno, sofre influência de diversos fatores estreitamente ligados, entre os quais a mãe merece destaque, por constituir o elemento fundamental nos cuidados com a criança, além da influência do contexto familiar, sócio-cultural e econômico. ${ }^{2}$ Os cuidados maternos exercem forte impacto sobre a saúde da criança e estão diretamente relacionados às informações em saúde, idade e grau de escolaridade da mãe, além do tempo dispensado a esses cuidados. ${ }^{3}$ Martin e Castillo ${ }^{4}$ referem que o ambiente social e familiar tende a valorizar o ganho ponderal da criança, considerando-o um indicativo de saúde, fato que contribui para um comportamento inseguro e ansioso da mãe, quanto ao ganho de peso da criança, interferindo no manejo alimentar da mesma. Vários estudos ${ }^{5-7}$ confirmam que o hábito alimentar inadequado da criança é responsável por diversos problemas de saúde, como obesidade, desnutrição e anemia.

Um suporte dos serviços de saúde dirigido a essas mães pode contribuir significativamente para melhorar a condução do processo alimentar dos filhos. Esta abordagem é efetivada a partir da consulta de enfermagem em puericultura, sendo o enfermeiro participante ativo desse processo. Pelo seu compromisso social de cuidar, educar e apoiar as mães e respectivas famílias, esse profissional está capacitado a fornecer orientações que poderão nortear o cuidado da mãe com a criança. ${ }^{8}$

É neste contexto que se insere o presente artigo, cujos objetivos foram: identificar os lactentes de cinco a oito meses que estão sendo alimentados segundo as orientações do Ministério da Saúde quanto à alimentação de crianças até o segundo ano de vida (crianças em aleitamento materno exclusivo, a partir do sexto mês deveriam receber três refeições diárias e crianças em aleitamento artificial cinco refeições ao dia), atendidos no Serviço de Puericultura do Instituto Materno Infantil Professor Fernando Figueira (IMIP); verificar o tipo de orientação recebida pelas mães, durante a consulta de enfermagem, para a realização da transição alimentar da criança, e detectar os principais problemas enfrentados pelas mães durante o processo de introdução da dieta de transição.

\section{Métodos}

Foi realizado um estudo descritivo exploratório desenvolvido no Instituto Materno Infantil Prof. Fernando Figueira (IMIP), na cidade do Recife, hospital de grande porte, referência para o atendimento da criança e da mulher. Nessa instituição funcionam programas de residência médica e de enfermagem, em várias especialidades, e Programa de Pós-graduação em Saúde Materno-Infantil. A área do estudo foi constituída pelo Serviço de Puericultura do Instituto Materno Infantil Prof. Fernando Figueira, no qual o atendimento foi realizado por duas enfermeiras especializadas na assistência à criança. A coleta de dados ocorreu no período de abril a agosto de 2003. Foram utilizados dados primários, procedentes da aplicação de um questionário às mães de crianças atendidas no serviço, pela própria pesquisadora.

Foram incluídas no estudo todas as mães de crianças na faixa etária entre cinco e oito meses $(n=101)$, em dieta de transição, atendidas no serviço de puericultura, acompanhando suas crianças no período do estudo.

Foram excluídas nove mães de crianças entre cinco e seis meses que, no momento da consulta, permaneciam em aleitamento materno exclusivo e, portanto, ainda não haviam iniciado a dieta de transição.

As variáveis estudadas foram as seguintes: caracterização geral da amostra (renda familiar em salários mínimos, chefe da família, número de pessoas na casa, tipo e regime de ocupação da casa, procedência da água, tipo de saneamento e destino do lixo); relacionadas à mãe (idade, escolaridade, atividade profissional e horas fora de casa, presença do companheiro, número de filhos e intervalo entre eles, ajuda para cuidar dos filhos e dos afazeres domésticos); relacionadas à criança: idade, peso ao nascer e atual, comprimento, tempo de aleitamento materno exclusivo, tipo de aleitamento no momento da transição alimentar; relacionadas ao ato de oferecer o alimento (pessoa que oferece os alimentos à criança, primeiro alimento oferecido na fase de tran- 
sição, freqüência e tipo de alimentos oferecidos, utensílios utilizados e dificuldades encontradas para o oferecimento dos alimentos); relacionadas à orientação (quem orientou a transição alimentar), se enfermeiro: tipo de orientação, (dúvidas após a orientação e oportunidade de esclarecimento durante a consulta).

Os dados foram coletados mediante aplicação de um questionário com 69 perguntas, sendo quatro abertas e 65 fechadas, constituído de cinco partes: caracterização da amostra, fatores relacionados à mãe, à criança, ao ato de oferecer o alimento e ao profissional de saúde. O instrumento de coleta foi previamente testado, através de estudo piloto, sendo aplicado para 15 mães de crianças atendidas na Puericultura do IMIP e no Serviço de Puericultura do Hospital das Clínicas.

No serviço de puericultura do IMIP as crianças em acompanhamento são agendadas para consulta mensalmente. As mães, ao comparecerem à consulta previamente agendada, eram solicitadas a participar, sendo-lhes explicada a importância e os objetivos do estudo. As mães que concordaram em participar assinaram o Termo de Consentimento Livre e Esclarecido, procedendo-se então à entrevista antes da consulta de puericultura com a enfermeira.

Os dados coletados foram introduzidos em um banco desenvolvido no software EPI-INFO, versão 6.0, em duas entradas distintas, e posteriormente interrelacionados através do comando VALIDATE, para verificação de erros. Posteriormente, esses dados foram analisados, tabulados em valores absolutos e percentuais e organizados em tabelas e gráficos.

Para classificação da dieta quanto à adequação às normas do Ministério da Saúde foram utilizadas duas categorias: crianças que recebiam leite materno devendo receber: três refeições/dia, sendo duas papas de sal e uma de fruta; e crianças em aleitamento misto ou artificial, devendo receber: cinco refeições/dia, sendo duas papas a partir de alimentos salgados (legumes, carne, feijão, frango, fígado/vísceras, etc), uma papa de fruta e dois mingaus (leite + amido/cereal).

O projeto de pesquisa foi aprovado pelo Comitê de Ética em Pesquisa com Seres Humanos do IMIP.

\section{Resultados}

Verificou-se que a maioria das mães $(66,3 \%)$ residia em casa própria, com abastecimento de água proveniente da rede pública $(90,1 \%)$, esgoto e fossa (respectivamente $49,5 \%$ e $38,6 \%$ ), lixo coletado (93,0\%), e a totalidade com luz elétrica. Os compa- nheiros das mães estudadas aparecem como os principais mantenedores das famílias $(71,3 \%)$, com renda variando entre um e dois salários mínimos em $60,3 \%$ da amostra (Tabela 1 ).

Em relação às características das mães (Tabela 2 ), observou-se que $80,2 \%$ delas eram jovens, com idades variando de 15 a 29 anos. O nível de escolaridade predominante foi acima de oito anos de estudo $(61,4 \%) ; 72,3 \%$ não exerciam atividade remunerada fora do lar; $52,5 \%$ eram primíparas e $58,4 \%$ recebiam ajuda no cuidado com seus filhos e nos afazeres domésticos.

Grande parte das mães do estudo proporcionou às suas crianças aleitamento materno exclusivo por um tempo superior ou igual a 120 dias (Tabela 3).

Em relação ao tempo de aleitamento materno

Tabela 1

Condições socioeconômicas das famílias de crianças de cinco a oito meses, em transição alimentar, atendidas em um serviço de puericultura. Recife, Pernambuco, 2003.

\begin{tabular}{|c|c|c|}
\hline Variáveis & $n=101$ & $\%$ \\
\hline \multicolumn{3}{|l|}{ Tipo de ocupação } \\
\hline Própria & 67 & 66,3 \\
\hline Alugada & 23 & 22,8 \\
\hline Outros & 11 & 10,9 \\
\hline \multicolumn{3}{|c|}{ Procedência da água } \\
\hline Rede pública & 91 & 90,1 \\
\hline Poço & 10 & 9,9 \\
\hline \multicolumn{3}{|l|}{ Saneamento } \\
\hline Esgoto & 50 & 49,5 \\
\hline Fossa & 39 & 38,6 \\
\hline Outros & 12 & 11,9 \\
\hline \multicolumn{3}{|l|}{ Lixo } \\
\hline Coletado & 94 & 93,0 \\
\hline Outros & 7 & 7,0 \\
\hline \multicolumn{3}{|l|}{ Luz elétrica } \\
\hline Sim & 101 & 100,0 \\
\hline \multicolumn{3}{|l|}{ Chefe da família } \\
\hline Pai & 72 & 71,3 \\
\hline Mãe & 5 & 5,0 \\
\hline Outros & 24 & 23,7 \\
\hline \multicolumn{3}{|l|}{ Renda } \\
\hline Sem renda & 5 & 5,0 \\
\hline 1 a 2 SM & 61 & 60,3 \\
\hline 3 a 5 SM & 25 & 24,8 \\
\hline 6 SM acima & 10 & 9,9 \\
\hline
\end{tabular}

$\mathrm{SM}=$ salário mínimo 
exclusivo, constatou-se que $67,3 \%$ das mães do estudo aproximaram-se das recomendações do Ministério da Saúde, ou seja, amamentar exclusivamente até o sexto mês. No entanto, vale ressaltar os $32,7 \%$ de mães que introduziram precocemente a prática de oferecer alimentos complementares à criança.

Quanto às particularidades relativas ao início da transição alimentar, a mãe, seguida da avó, foram as pessoas mais presentes na oferta dos alimentos às crianças. Na transição alimentar, o suco foi o primeiro alimento a ser oferecido à criança $(69,3 \%)$ (Tabela 4).

O Gráfico 1 mostra a situação da transição alimentar das crianças quanto às recomendações do Ministério da Saúde.

Conforme os resultados mostrados, constatou-se que uma parcela significativa de crianças $(79,2 \%)$ não teve uma transição alimentar adequada às orientações

Tabela 2

Perfil das mães de crianças de cinco a oito meses, em dieta de transição, atendidas em um serviço de puericultura. Recife, Pernambuco, 2003.

\begin{tabular}{lcc}
\hline Variáveis & $\mathrm{n}=101$ & $\%$ \\
\hline Faixa etária & & 81 \\
15 a 29 anos & 20 & 80,2 \\
$>30$ anos & 39 & 19,8 \\
Escolaridade & 62 & 38,6 \\
$<8$ anos de estudo & & 61,4 \\
$\geq 8$ anos de estudo & 28 & 27,7 \\
Trabalho fora do lar & 73 & 72,3 \\
Sim & & \\
Não & 53 & 52,5 \\
Paridade & 27 & 26,7 \\
1 & 16 & 15,8 \\
2 & 5 & 5,0 \\
3 & & 58,4 \\
$>4$ & 59 & 41,6 \\
Ajuda no cuidado com os filhos/afazeres domésticos & 42 & \\
Nim & & \\
\hline
\end{tabular}

\section{Tabela 3}

Tempo de aleitamento materno exclusivo de crianças de cinco a oito meses, em dieta de transição, atendidas em um serviço de puericultura. Recife, Pernambuco, 2003.

\begin{tabular}{lrr}
\hline Tempo & $\mathbf{n}$ & $\%$ \\
\hline$<30$ dias & 3 & 3,0 \\
De 30 a 60 dias & 20 & 19,8 \\
Até 90 dias & 10 & 9,9 \\
$\geq 120$ dias & 68 & 67,3 \\
Total & 101 & 100,0 \\
\hline
\end{tabular}

Tabela 4

Particularidades referentes à dieta de transição de crianças de cinco a oito meses, atendidas em um serviço de puericultura. Recife, Pernambuco, 2003.

\begin{tabular}{lcc}
\hline Particularidades & $\mathbf{n = 1 0 1}$ & $\%$ \\
\hline Quem oferece os alimentos & 73 & 72,3 \\
Mãe & 17 & 16,8 \\
Avó & 3 & 3,0 \\
Tia & 1 & 1,0 \\
Pai & 7 & 6,9 \\
Outros & & \\
Primeiro alimento oferecido & 70 & 69,3 \\
Suco & 16 & 15,8 \\
Papa de frutas & 15 & 14,9 \\
Sopinha & & \\
\hline
\end{tabular}


preconizadas pelo Ministério da Saúde.

$\mathrm{Na}$ Tabela 5 estão explicitadas as razões pelas quais as mães não seguem as orientações transmitidas no serviço, quanto à dieta de transição. Verificou-se que os principais motivos mencionados pelas mães para justificar o não seguimento das normas preconizadas pelo Ministério da Saúde para a transição alimentar foram: a não aceitação da criança $(25,3 \%)$, a interferência da avó $(19,0 \%)$ e a comodidade do preparo/oferecimento do mingau em relação à alimentação salgada $(17,7 \%)$.

A Tabela 6 revela a percepção das mães acerca

\section{Gráfico 1}

Adequação às normas de transição alimentar do Ministério da Saúde de crianças de cinco a oito meses, atendidas num serviço de puericultura. Recife, Pernambuco, 2003.

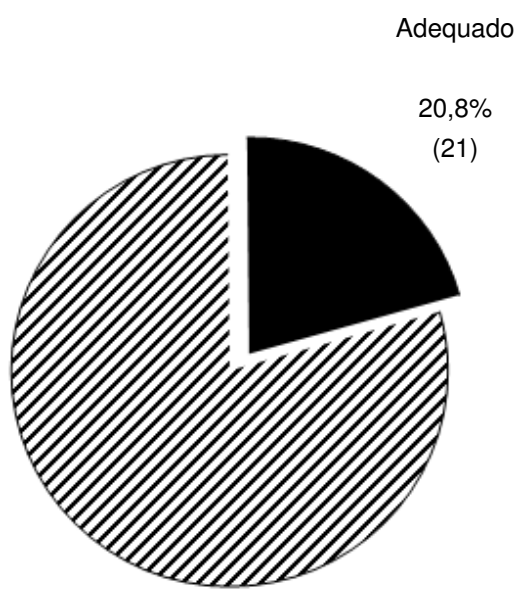

Inadequado

$79,2 \%$

(80) da qualidade das orientações fornecidas pelo enfermeiro, com relação à dieta de transição. Para a maioria (70,5\%), o enfermeiro ressaltou a importância dos alimentos complementares oferecidos à criança. Observou-se que $97,4 \%$ das mães esclareceram suas dúvidas e receberam orientações por escrito, além das verbais, e 94,8\% não tinham dúvidas, após a consulta. Apenas 2,6\% das mães afirmaram não ter tido oportunidade de esclarecer dúvidas, durante a consulta.

Tabela 5

Razões alegadas por 80 mães de crianças de cinco a oito meses que não seguiam as normas do Ministério da Saúde transmitidas no serviço para transição alimentar, atendidas em um serviço de puericultura, quanto à dieta de transição. Recife, 2003.

\begin{tabular}{lrr}
\hline Motivos & $\mathbf{n = 7 9}$ & $\%$ \\
\hline Porque a criança não aceita & 20 & 25,3 \\
Falta do alimento & 12 & 15,2 \\
Dar o leite materno é mais fácil & 12 & 15,2 \\
Porque a avó não concorda & 15 & 19,0 \\
Não tem tempo para preparar o alimento & 6 & 7,6 \\
Preparar/dar o mingau é mais fácil que o alimento salgado & 14 & 17,7 \\
\hline
\end{tabular}

* Uma mãe que não se adequava às normas de transição alimentar do Ministério da Saúde não declarou razão.

\section{Tabela 6}

Percepção das mães de crianças de cinco a oito meses, atendidas em um serviço de puericultura, com relação à qualidade das orientações recebidas, na consulta de enfermagem, para a transição alimentar de seus filhos. Recife, Pernambuco, 2003.

\begin{tabular}{lcc}
\hline Percepção & $\mathbf{n}=\mathbf{7 8}$ & $\%$ \\
\hline O enfermeiro ressaltou a importância dos alimentos & & \\
complementares para a criança & 55 & 70,5 \\
Sim & 23 & 29,5 \\
Não & \\
Houve dúvidas após a orientação & 4 & 5,2 \\
Sim & 74 & 94,8 \\
Não & 76 & 97,4 \\
Teve oportunidade de esclarecer as dúvidas durante a consulta & 2,6 \\
Sim & 2 & \\
Não & 76 & 2,6 \\
Recebeu orientações por escrito, além das verbais & 2 & \\
Sim & & \\
Não & & \\
\hline * Total de mães orientadas pelo enfermeiro, com relação à transição alimentar da criança; \\
as demais receberam orientação de outros profissionais ou de pessoas da família para \\
transição alimentar das crianças.
\end{tabular}




\section{Discussão}

Ao se traçar o perfil socioeconômico das famílias das mães entrevistadas, foi constatado que a maioria sobrevivia com dois salários mínimos, sendo o pai o principal mantenedor. Grande parte possuia casa própria, servida por luz elétrica, e um número significativo de moradias dispunha de água encanada e adequado saneamento; observou-se também a predominância do destino correto do lixo.

Considerando que o processo de alimentação está intimamente relacionado com a presença e reciprocidade de emoções diversas, o ambiente onde a criança é alimentada deve ser entendido como agente de grande importância. Porém, garantir condições ambientais adequadas para essa prática nem sempre é uma tarefa simples para boa parte da população. Más condições de habitação e de higiene, bem como carência econômica, podem constituir fatores de influência negativa sobre a saúde da criança. ${ }^{9}$ Dessa forma, ao se deparar com uma criança que não esteja crescendo e se desenvolvendo normalmente, devem ser investigados os seus hábitos alimentares, as condições econômicas e sociais da família em que vive, bem como a maneira como é cuidada. ${ }^{10}$

Sob este enfoque, a mãe se destaca como principal agente cuidador da criança. No estudo, as mães, em sua maioria, caracterizavam-se como adultas jovens e primíparas, grande parte não exercia atividade remunerada e quase metade recebia ajuda nos afazeres domésticos e no cuidado com os filhos. No tocante à escolaridade, a maioria tinha escolaridade acima de oito anos de estudo. Tem sido referido que, dependendo da idade e do grau de escolaridade materna, do acesso às informações em saúde e do tempo dedicado aos cuidados com a criança, essa tem um maior ou menor risco de adoecer. ${ }^{3}$ Vale salientar, também, que a primiparidade é um determinante de insegurança para as mães, gerando conflitos, pois acentua as dificuldades em tomar decisões sobre seguir as orientações dos profissionais de saúde ou aceitar conselhos das pessoas mais velhas nos cuidados com a criança. ${ }^{8-13}$

A avó também se destacou como pessoa responsável pelo cuidado com a criança. Primo e Caetano ${ }^{12}$ referem que a filha se espelha na mãe e vislumbra-a como modelo de vida a ser seguido, copiado e transformado. Dessa forma, o componente educacional familiar favorece a alimentação e o cuidado com a criança, quando a filha passa a espelhar sua vida e seu comportamento na forma como sua mãe foi criada. Porém, foi observado, no presente estudo, que a influência da avó não foi tão positiva, como será discutido adiante.
Entre os cuidados essenciais à saúde da criança está o aleitamento materno. O tempo de aleitamento materno exclusivo referido pelas mães foi superior ou igual a 120 dias, aproximando-se das orientações da Organização Mundial de Saúde (OMS). Contudo, foi relevante o número de crianças introduzidas na alimentação complementar precocemente.

A alimentação complementar somente deveria ser iniciada a partir do sexto mês de idade, gradativamente, sob a forma de purês de legumes, frutas, carnes e cereais. ${ }^{14-16}$ Para o início da transição alimentar a grande maioria das mães utilizou o suco como primeiro alimento a ser oferecido, o que pode significar um reflexo das orientações anteriores de condutas alimentares segundo o Ministério da Saúde $^{14}$ (que orientava o oferecimento do suco pela manhã, como uma refeição), repassadas anteriormente neste mesmo serviço, local do estudo. Possivelmente, justificando a prática acima, as mães não primíparas vivenciaram a experiência da transição alimentar com filhos mais velhos podem ter seguido essa orientação anterior. Durante a transição a mãe foi a principal responsável pela oferta dos alimentos à criança, seguida da avó.

Entretanto, a transição alimentar não ocorreu de maneira adequada, uma vez que $79,2 \%$ das crianças do estudo não se encontravam alimentadas de acordo com as recomendações do Ministério da Saúde. ${ }^{14}$ Como consequiência de um padrão alimentar inadequado podem ocorrer problemas, como anemia ferropriva, conforme afirmam Queiroz e Torres. $^{7}$ Possivelmente, como reflexo de uma alimentação inadequada, um estudo realizado com população semelhante, no ambulatório de pediatria do IMIP, encontrou um alto percentual de anemia em crianças de seis a vinte e três meses. ${ }^{17}$ Os principais motivos pelos quais as mães não se adequavam às orientações do Ministério da Saúde sobre transição alimentar foram a não aceitação da criança, a interferência da avó e a comodidade do preparo do mingau em relação aos alimentos salgados ${ }^{14}$. Entende-se com isso que ocorre desistência da mãe/cuidador quando a criança rejeita a alimentação, o que prejudica o processo de condicionamento; ou seja, para que um alimento seja aceito, não pode ser apenas percebido visualmente ou pelo odor, é necessário que a criança prove o alimento, mesmo que inicialmente em quantidade mínima, para que esse condicionamento se produza. $^{15-18}$

Geralmente, a aceitação de um novo alimento ocorre somente após 12 a 15 apresentações do mesmo, podendo haver desistência dos pais nesse período, por achar que a criança não gosta do alimento. ${ }^{15-18}$ Monte e Sá. ${ }^{18}$ afirmam que as 
atribuições da mãe no lar e os afazeres domésticos são fatores que interferem na prática alimentar das crianças. No presente estudo, as mães citaram como um dos motivos para o não oferecimento do alimento salgado a praticidade no preparo do mingau, embora a maioria tenha afirmado que recebe ajuda para cuidar dos filhos e das atividades domésticas. A principal inadequação da dieta ocorreu pelo não oferecimento da segunda refeição salgada, sendo nesse horário oferecido um mingau à criança. Osório, ${ }^{19}$ em um estudo sobre anemia, observou que as mães, geralmente, substituem uma refeição pela mamadeira, o que contribui para a presença de anemia.

Várias pesquisas sustentam que o apoio e o suporte familiar, principalmente do pai e da avó, são fatores importantes na escolha da alimentação da criança. ${ }^{20}$ No presente estudo foi constatado que a avó participa efetivamente da educação alimentar das crianças. Para introduzir a alimentação complementar é de fundamental importância receber orientações adequadas, além do devido acompanhamento das crianças por um profissional de saúde.

Quanto às orientações fornecidas à mãe durante o processo de transição, grande parte das orientações oferecidas pelo enfermeiro ressaltou a importância dos alimentos. Um estudo ${ }^{21}$ realizado em populações cubanas e porto-riquenhas sobre práticas de alimentação infantil apontou a avó materna como a mais importante fonte de informações seguidas pelas mães, em detrimento dos profissionais de saúde. Com isso, as avós repassam suas crenças e valores ao processo de alimentação da criança.

As orientações, em sua quase totalidade, foram repassadas por escrito, além de verbalizadas. Um pequeno percentual de mães referiu dúvidas após a consulta e não ter tido oportunidade de esclarecê-las. Esses resultados corroboram com a opinião de Silva, ${ }^{22}$ ao enfatizar que, para que haja uma comunicação efetiva, se faz necessária a existência de duas pessoas ou mais, em proximidade física, e que é necessário que essas pessoas percebam a presença uma da outra, haja interdependência comunicativa, troca de mensagens e compreensão de mensagens verbais e não-verbais. Durante a consulta de enfermagem ocorre uma interação face a face entre as pessoas. O enfermeiro e o cliente estabelecem e mantêm uma relação definida por percepções mútuas. 23

Os relatos das mães em relação às orientações durante a consulta de enfermagem não condizem com o percentual de inadequação à dieta encontrado no estudo, mesmo em se tratando de um serviço em que as orientações repassadas seguem as recomendações preconizadas pelo Ministério da Saúde. É possível que os tabus da família e as decisões da avó/cuidador estejam direcionando o padrão alimentar da criança, sobressaindo-se às orientações recebidas pelas mães na consulta de enfermagem. Não se pode também descartar a possibilidade de que essas orientações, mesmo normatizadas, não estejam sendo repassadas às mães de maneira que elas as absorvam e tenham condições de praticá-las.

A mãe foi o principal executor dos cuidados e receptor das orientações fornecidas pelo enfermeiro. Elas mostraram, segundo os dados, que houve uma boa qualidade da consulta de enfermagem. No entanto, os resultados não apontaram para uma adequação às normas do Ministério da Saúde. Diante desse cenário pergunta-se então: o que estava acontecendo de errado?

Scotney ${ }^{24}$ considera que durante o processo de comunicação poderá ocorrer que: as pessoas ouvem, mas não compreendem; ouvem e pensam que compreendem, não executando as orientações de maneira certa ou transmitindo a outrem de maneira errada; ouvem e compreendem, mas não ficam convencidas e não modificam seus hábitos. Dessa maneira a comunicação será considerada ineficaz.

Ocorre o insucesso da educação em saúde quando o educador não consegue comunicar-se de maneira eficaz, não refletindo os resultados esperados. Para o êxito da comunicação em saúde, há de se considerar que: as pessoas são diferentes e precisam ser tratadas de maneira diferente; o processo de educação em saúde deve acompanhar as mudanças do contexto social em questão; deve-se perceber a necessidade de um trabalho em equipe; e deve haver um planejamento das ações com avaliação dos resultados. ${ }^{25}$

Outro aspecto relevante nesse processo de comunicação é o tipo de linguagem utilizada, por exemplo, a linguagem não-verbal, que não se faz só com a palavra falada, mas também através de sinais, expressão corporal e através da escrita. ${ }^{25}$ Durante o estudo foi observado que as mães informaram ter recebido orientações escritas, além das orientações faladas. Porém, no caso das mães que referiram baixo nível de escolaridade esse artifício pode não ter sido eficaz.

A comunicação torna-se satisfatória quando ela é capaz de modificar comportamentos em saúde, significa ainda que uma mensagem foi enviada e recebida. Sendo assim, é um processo que envolve aquele que recebe a mensagem e aquele que a envia, em um processo mútuo de idas e vindas, ações e reações. ${ }^{25}$

Assim, não se pode atribuir o insucesso do não entendimento ou apreensão das mensagens oferecidas pelo enfermeiro à mãe/cuidador, pois, como foi mencionado, a comunicação não é determinada por 
apenas uma das partes envolvidas. A educação para saúde só é coroada de êxito quando os esforços planejados alteram o comportamento das pessoas em prol da saúde. ${ }^{25}$

O presente estudo, por seu caráter descritivo exploratório, apresenta algumas limitações, não sendo suficiente para responder algumas questões sobre o assunto abordado, pois existem outros fatores, relacionados à alimentação da criança, que não foram focados no trabalho. $\mathrm{O}$ estudo deu ênfase à mãe, pela sua magnitude no cuidado com a criança. Desse modo, é evidente a necessidade de outros estudos que

\section{Referências}

1. Arantes CIS. Amamentação: visão das mulheres que amamentam. J Pediatr (Rio J). 1995; 71: 195-202.

2. Ctenas MLB, Vitolo MR. Crescendo com saúde: o guia de crescimento da criança. São Paulo: C2 Ed. e Consultoria em Nutrição; 1999.

3. Silva GAP. O uso de chupeta contribui para uma maior ocorrência de enteroparasitoses? J Pediatr (Rio J). 1997; 73: 88-91.

4. Martin VB, Castillo CD. El niño que no quiere comer. Rev Chilena Pediatr. 2000; 71: 139-41.

5. Escravidão MAMS, Vieira GO. Obesidade exógena na infância e na adolescência. J Pediatr (Rio J). 2000; 76 (Supl. 3): 104-7.

6. Monteiro CA, Freitas ICM. Tendência secular da anemia na infância na cidade de São Paulo (1984-1996). Rev Saúde Pública. 2000; 34 (Supl. 6): 62-72.

7. Queiroz SS, Torres MAA. Anemia ferropriva na infância. J Pediatr (Rio J). 2000; 76 (Supl. 3) : S298-S304.

8. Souza MCC. Compreendendo a ação materna frente às orientações sobre a alimentação da criança de 0 a 6 meses [dissertação mestrado] Belo Horizonte: Escola de Enfermagem da Universidade Federal de Minas Gerais; 2001.

9. Ricco RG, Del Ciampo LA, Almeida CAN. Puericultura: princípios e práticas: atenção integral à saúde da criança. São Paulo: Atheneu; 2000.

10. Leão E, Corrêa EJ, Viana MB, Mota JA. Pediatria ambulatorial. Belo Horizonte: COOPMED; 1998.

11. Uebel WS. Contribuição ao estudo dos problemas da criança de 0 a 12 meses: diagnosticados na primeira consulta de enfermagem [dissertação mestrado]. Rio de Janeiro: Universidade Federal do Rio de Janeiro; 1984.

12. Primo CC, Caetano LC. A decisão de amamentar da nutriz: percepção de sua mãe. J Pediatr (Rio J). 1999; 75: 449-55.

13. Brasil. Ministério da Saúde. Guia alimentar para crianças menores de 2 anos. Brasília, DF; 2002.

14. Giugliani ERJ, Victora CG. Normas alimentares para crianças brasileiras menores de 2 anos: bases científicas. Brasília, DF: Organização Pan-Americana da Saúde, Organização Mundial de Saúde; 1997. visem abranger e explicar aspectos não abordados nesse trabalho.

É importante referir a possível ocorrência de um viés de aferição, considerando ter sido a mesma pessoa, no caso a pesquisadora, responsável pela aplicação dos questionários e pelas consultas de enfermagem no serviço. As mães podem ter se sentido "pouco à vontade" para relatar suas dificuldades e problemas de comunicação durante a consulta, fato que repercutiria nos resultados relativos à qualidade das orientações fornecidas pelos enfermeiros do serviço.
15. Brasil. Ministério da Saúde. Atenção integrada às doenças prevalentes na infância. Brasília, DF; 2003.

16. Leal LP. Reprodutibilidade de sinais clínicos e da escala de cores da hemoglobina da OMS no diagnóstico de anemia em crianças [dissertação mestrado]. Recife: Departamento de Nutrição da Universidade Federal de Pernambuco; 2002.

17. Stein LM, Ramos M. Desenvolvimento do comportamento alimentar infantil. J Pediatr (Rio J). 2000; 76 (Supl. 3): S229-S37.

18. Monte CMC, Sá MLB. Promoção de nutrição de crianças menores de cinco anos no dia-a-dia da comunidade: manual para médicos, enfermeiros, nutricionistas e agentes comunitários de saúde. Vitória; 2001.

19. Osório MM. Fatores determinantes para anemia em crianças. J Pediatr (Rio J). 2002; 78: 269-78.

20. Vaahtera M, Nagy E, Orvos H. Breastfeeding and complementary feeding pratices in rural malawi. Acta Pediatr. 2001; 90: 228-332.

21. Bryant CD. The impact of Kin, friend and neighbor networks of infant feeding pratices. Soc Sci Med. 1982; 16: 1757-65.

22. Silva MG. A consulta de enfermagem no contexto da comunicação interpessoal: a percepção do cliente. Rev LatinoAmer Enferm. 1998; 6: 56-9.

23. Silva MJP. A enfermagem frente à necessidade de percepção do paciente. Rev Paul Enferm. 1990;9: 42-5.

24. Scotney N. Educação para a saúde: manual para o pessoal da zona rural. São Paulo: Paulinas; 1991.

25. Arruda BKG. A educação profissional em saúde e realidade social. Recife: IMIP; 2001 (Publicações Científicas do Instituto Materno Infantil de Pernambuco).

Recebido em 2 de setembro de 2006

Versão final apresentada em 30 de maio de 2007

Aprovado em 13 de julho de 2007 